\title{
Ernst Vogt \\ Das Werk August Böckhs als Herausforderung für unsere Zeit
}

Am heutigen Tage jährt sich zum 212. Male der Geburtstag von August Böckh. ${ }^{1}$ Lassen Sie uns für einen Augenblick zurückblicken in das Jahr 1785: Europa steht am Vorabend der Französischen Revolution, und in dem Prozeß um die Halsbandaffäre wird zeichenhaft die Schwäche des Ancien Régime sichtbar. Friedrich der Große schließt, ein Jahr vor seinem Tode, mit den Kurfürsten von Sachsen und Hannover den Deutschen Fürstenbund, der den Versuch des Hauses Habsburg verhindern soll, Bayern gegen die österreichischen Niederlande einzutauschen. In Preußen arbeitet die erste deutsche Dampfmaschine, der Ärmelkanal wird im Freiballon überquert, und in England wird die 'Times' gegründet. Der neunundzwanzigjährige Wolfgang Amadeus Mozart arbeitet an seiner Oper 'Figaros Hochzeit' und schreibt das Klavierkonzert in d-moll. Auf dem Buchmarkt erscheinen Immanuel Kants 'Grundlegung zur Metaphysik der Sitten' und Friedrich Heinrich Jacobis Schrift 'Über die Lehre des Spinoza, in Briefen an Moses Mendelssohn', in der Goethes Gedichte 'Edel sei der Mensch, hilfreich und gut' und 'Prometheus' erstmals abgedruckt sind; es erscheint das erste Heft der Rheinischen Thalia mit Schillers Mannheimer Rede 'Was kann eine gute stehende Schaubühne wirken?' (später bekannt unter dem Titel 'Die Schaubühne als eine moralische Anstalt betrachtet'), es erscheinen der erste Teil des autobiographischen Romans 'Anton Reiser' von Karl Philipp Moritz und die 'Gedichte aus dem Kerker' des zehn Jahre auf der Festung Hohenasperg gefangen gehaltenen Dichters Christian Friedrich Daniel Schubart, des Verfassers des Kapliedes.

Dies ist die Welt, in die August Böckh am 24. November 1785 in Karlsruhe als jüngstes von sechs Kindern hineingeboren wird. Eine Schwester Schubarts war mit dem Nördlinger Pfarrer und Archidiakon Christian Gottfried Böckh verheiratet, und dessen jüngerer Bruder, der Hofratssekretär und kaiserliche Notar Georg 
Matthäus Böckh in Karlsruhe, ist der Vater von August Böckh. Nach dem Tode des Vaters, der bereits 1790 starb, gestaltete sich das Leben für die Familie nicht einfach - ,ein Kind einer Familie, die von schweren Schlägen des Schicksals niedergebeugt war" hat Böckh sich später einmal genannt ${ }^{2}$-, doch konnte er nach dem Besuch des Karlsruher Gymnasiums, an dem unter anderen Johann Peter Hebel sein Lehrer war, 1803 in Halle das Studium der evangelischen Theologie aufnehmen. Hier wurde er freilich schon bald von Friedrich August Wolf, dem Schüler Christian Gottlob Heynes und bedeutendsten Altertumswissenschaftler seiner Zeit, für die Philologie gewonnen, empfing starke Anregungen aber zugleich von den Platonvorlesungen des 1804 nach Halle berufenen Schleiermacher. Auf die Studienzeit folgte vom Frühjahr 1806 bis Anfang 1807 eine erste Begegnung mit Berlin: Ausbildung am Seminar für gelehrte Schulen, Unterricht am Gymnasium zum Grauen Kloster, Privatstunden, wobei u. a. der junge Meyerbeer (1791-1864) sein Schüler war, und, gemeinsam mit Buttmann, Heindorf, Spalding und anderen, Tätigkeit in der Griechischen Gesellschaft. Nach der Doppelschlacht von Jena und Auerstedt und dem Einmarsch der Franzosen in Berlin strebte Böckh jedoch die Rückkehr in seine badische Heimat an und erhielt noch im Jahre 1807, zweiundzwanzigjährig, eine außerordentliche Professur an der Universität Heidelberg, die 1809, nach Ablehnung eines Rufes an die Universität Königsberg, in ein Ordinariat umgewandelt wurde. Durch seinen nächsten Kollegen Friedrich Creuzer gewann er in diesen Jahren auch Verbindung zu dem in Heidelberg wirkenden Kreis der Romantiker um Achim von Arnim und Clemens Brentano.

Im Herbst 1810 erhielt Böckh einen Ruf an die neugegründete Universität Berlin, den er zum Sommersemester 1811 annahm. Damit begann hier in dieser Stadt eine Wirksamkeit, die über mehr als sechsundfünfzig Jahre bis zu seinem Tode am 3. August 1867 angedauert hat. Sechsmal wurde er zum Dekan seiner Fakultät gewählt, fünfmal zum Rektor, zuletzt als beinahe Fünfund siebzigjähriger im Amtsjahr 1859/60, dem Jahr, in das die 50-Jahr-Feier der Berliner Universität fiel. So hat es seine tiefe Berechtigung, daß die Humboldt-Universität ihr neues Instituts- 
gebäude nach Böckh als einem ihrer bedeutendsten und verdientesten Mitglieder benannt hat. Seit 1814 gehörte Böckh auch der Königlich Preußischen Akademie der Wissenschaften an, deren philosophisch-historischer Klasse er als Nachfolger Schleiermachers siebenundzwanzig Jahre hindurch, von 1834 bis 1861, als Sekretar vorgestanden hat.

Das wissenschaftliche Werk, das in diesen Jahrzehnten allmählich heranwuchs, sucht an Umfang und Gewicht seinesgleichen: die frühen Platonstudien, die vor allem für das Verständnis der Metrik bahnbrechende Pindarausgabe mit Kommentar, die monumentale 'Staatshaushaltung der Athener', die Inschriftensammlung des Corpus Inscriptionum Graecarum, zahlreiche weitere Monographien und Abhandlungen, die sieben Bände der Kleinen Schriften und die erst nach Böckhs Tode veröffentlichte 'Encyklopädie und Methodologie der philologischen Wissenschaften'. Eine zureichende Würdigung dieses umfassenden Werkes ist im Rahmen unserer heutigen Veranstaltung natürlich ganz unmöglich. Lassen Sie mich stattdessen einige Aspekte dieses Werkes herausstellen, mit denen es weit in die Zukunft vorausgewiesen hat. Denn dieses Werk hat Maßstäbe gesetzt, denen auch wir heute uns noch zu stellen haben. Und das gilt nicht nur für die Altertumswissenschaft als diejenige Disziplin, in der Böckh tätig gewesen ist und in der er seine großen, bahnbrechenden Leistungen vollbracht hat, sondern für die Wissenschaft schlechthin im Sinne von Max Webers Schrift 'Wissenschaft als Beruf' und darüber hinaus für die Wissenschaft als einen Teil der geistigen Existenz des Menschen überhaupt.

Sucht man in dieser Weise die weit in die Zukunft hineinreichende Bedeutung des Böckhschen Lebenswerkes zu bestimmen, so kann es in mehrfacher Hinsicht als beispielhaft gelten: zunächst als ein Paradigma fachübergreifender Forschung, in dem die Einzelerscheinung mit wachem Sinn für das Ganze in einen größeren Zusammenhang gestellt ist. Sodann in seinem klaren Methodenbewußtsein, das die Prinzipien ebenso wie die einzelnen Schritte wissenschaftlicher Beweisführung in ungewöhnlicher Weise reflektiert. Weiter in seinem offenen Bekenntnis zu der 
Verantwortung des Wissenschaftlers vor der Öffentlichkeit, das von selbstgenügsamer Einzelforschung ebensoweit entfernt ist wie von eilfertiger Anpassung an die Tendenzen der Zeit. Und schließlich in der aus tiefer Einsicht gewonnenen Überzeugung von der Bedeutung des griechisch-römischen Altertums für das Leben der Gegenwart. Ich will versuchen, Ihnen das jeweils an einigen Beispielen zu verdeutlichen.

Der fachübergreifende (heute würden wir sagen: interdisziplinäre) Aspekt des Böckhschen Werkes hat seine Wurzeln in der ebenso breiten wie gründlichen Ausbildung auf dem Gymnasium in Karlsruhe, wo Böckh nicht nur in den alten Sprachen, sondern etwa auch in der Botanik und vor allem in der Mathematik einen vorzüglichen Unterricht erhielt. Weitere Förderung erfuhr diese Breite der Interessen durch Böckhs akademischen Lehrer Friedrich August Wolf. War die Philologie bis in die zweite Hälfte des 18. Jahrhunderts hinein im wesentlichen Hilfsdisziplin der Theologie, der Philosophie und der Rechtswissenschaft gewesen, so hatte der junge Wolf sich 1777 an der Universität Göttingen selbstbewußt als 'studiosus philologiae' einschreiben lassen und entwickelte ab 1783 in seinen Vorlesungen an der Universität Halle seine Konzeption einer umfassenden, auf die möglichst vollständige Kenntnis des gesamten Lebens der Griechen und Römer gerichteten Altertumswissenschaft. Schon in Böckhs früher, 1807 veröffentlichter Abhandlung 'Über die Bildung der Weltseele im Timaeos des Platon', in der philologische, philosophische, musikalische und mathematische Kenntnisse in gleicher Weise zur Geltung kommen, tritt diese Weite des Horizontes in Erscheinung. Gerade seine Ausbildung und Bildung auf musikalischem Gebiete waren es auch, die es Böckh ermöglichten, sich fruchtbar und ergebnisreich mit den schwierigen Problemen der griechischen Musik und Metrik auseinanderzusetzen. Als ein erster Ertrag dieser Bemühungen erschien 1809 seine Abhandlung 'Über die Versmaße Pindars'. 1811 begann dann seine große Pindarausgabe zu erscheinen, die zehn Jahre später, im Jahre 1821, mit dem 2. Teil des 2. Bandes abgeschlossen vorlag und die das Verständnis dieses größten griechischen Chordichters revolutioniert hat. Der griechische Text war, auf die Auswertung der Handschriften sowie auf die kritische Ar- 
beit von Generationen von Gelehrten gestützt, auf eine neue Grundlage gestellt, dem richtigen Verständnis des metrischen Aufbaus der einzelnen Chorlieder die Bahn gebrochen, das antike Erklärungsmaterial, die sogenannten Scholien, vollständig vorgelegt und ausgewertet und das Ganze in einem umfassenden Kommentar, an dem für die nemeischen und isthmischen Lieder Böckhs Freund Ludolph Dissen in Göttingen beteiligt war, in seinem historischen Kontext allseitig erläutert. Mit vollem Recht durfte Böckh auf dem Titelblatt seines Werkes darauf hinweisen, in seinem Texte die ursprünglichen Metra wiederhergestellt zu haben (,textum in genuina metra restituit"). Seine in scharfsinniger Analyse gewonnene Verseinteilung und Verszählung haben sich gegenüber der älteren von Christian Gottlob Heyne durchgesetzt und bestimmen den Pindartext bis hin zu der heute maßgebenden Ausgabe von Bruno Snell und Herwig Maehler.

Das Werk jedoch, in dem das Zusammenwirken verschiedener Disziplinen und der Blick auf das Ganze zu den bedeutendsten Ergebnissen führen, ist zweifellos Böckhs 'Staatshaushaltung der Athener'. Sie bietet, wie Thomas Nipperdey es formuliert hat, ,ein Bild der griechischen Welt jenseits des Ästhetischen und Religiösen wie des Nur-Politischen, ein Stück Sozial- und Wirtschaftsgeschichte aus dem klassisch-romantischen Geist der 'Totalität', der Einheit und des Zusammenhangs einer menschlichgeschichtlichen Welt".

In diesem bahnbrechenden Werk ist die damals noch in ihren Anfängen steckende Inschriftenforschung zum ersten Male aus ihrer Isolierung gelöst und in den Dienst einer großen, umfassenden Aufgabe gestellt, die literarische ebenso wie die monumentale Überlieferung mustergültig aufgearbeitet, der gewaltige Stoff in souveränem Weitblick dargeboten. Auf die Behandlung der Preise, Löhne und Zinsen in Attika folgt die Darstellung der Finanzverwaltung und der Ausgaben, der ordentlichen wie der außerordentlichen Einkünfte und der besonderen Finanzmaßregeln des Athenischen Staates. 
Böckh geht von den Edelmetallen Silber und Gold als dem Maßstab der Preise und von dem Verhältnis ihres Wertes zueinander aus und entwirft zunächst ein außerordentlich farbenreiches Panorama von Zahl und Gliederung der attischen Bevölkerung, ihrer Tätigkeit in Landwirtschaft, Gewerbe und Handel, von den Besitzverhältnissen hinsichtlich Häusern, Sklaven und Vieh, von der Produktion und Verteilung der wichtigsten Nahrungsmittel, den Mahlzeiten, der Kleidung, der Herstellung von Geräten, Waffen und Schiffen und vor allem von den für den Lebensunterhalt erforderlichen Summen sowie von deren Verhältnis zum Volksvermögen und zu den gezahlten Löhnen. Immer wieder werden dabei aufschlußreiche Parallelen zu den zeitgenössischen Verhältnissen (etwa auf den Zuckerrohrplantagen in Amerika) gezogen, über die Böckh eine bemerkenswerte Kenntnis zeigt. Mit welch wachem sozialen Gewissen Böckh seinen Gegenstand behandelte, mag etwa sein Urteil belegen, die Phokier hätten einem Käufer von tausend Sklaven namens Mnason, ,nicht mit Unrecht“ so seine Wertung - vorgeworfen, er nehme damit ebensovielen Bürgern die notwendige Nahrungsgrundlage weg. ${ }^{4}$

Des weiteren geht es dann, mit ständigem Blick auf die Finanzverwaltung der Neuzeit, um die Frage, in welcher Weise das Finanzwesen im Altertum und insbesondere die attische Staatshaushaltung organisiert waren. Dazu werden die für die Finanzgesetzgebung und die Finanzverwaltung zuständigen Behörden auf Grund aller uns verfügbaren Quellen im einzelnen beschrieben und in ihren jeweiligen Funktionen dargestellt, sodann die vielfältigen Ausgaben der Polis, nicht zuletzt diejenigen zur Unterstützung der Armen und Gebrechlichen, detailliert behandelt. Mitunter muten die Ausführungen Böckhs wie indirekte Forderungen nach einer Sozialgesetzgebung an, wie sie erst die 2. Hälfte des 19. Jahrhunderts gebracht hat, etwa wenn es über das Athen des 5. Jahrhunderts v. Chr. heißt: „Eine löbliche Anstalt war die Unterstützung der Bürger, welche wegen körperlicher Gebrechen oder Schwäche ihren Unterhalt zu erwerben unfähig waren“.5

Böckh geht anschließend zu der Behandlung der ordentlichen, d.h. der regelmäßigen Einkünfte der attischen Polis über und klärt 
nacheinander die verschiedenen Zölle (Einfuhr-, Ausfuhr-, Hafen- und Seezölle), die Gewerbe- und Personensteuern, Strafgelder, Tribute und Leiturgien. Der letzte Teil des Werkes ist schließlich den außerordentlichen Einkünften des Athenischen Staates und den besonderen Finanzmaßregeln gewidmet, die vor allem im Falle eines Krieges Anwendung fanden.

So sehr die Einzelforschung in diesem großen Werke allenthalben zu ihrem Rechte kommt, so wenig ist doch je der historische Rahmen aus dem Blick verloren, entsprechend den Grundsätzen, die Böckh in den 'Vorerinnerungen zur ersten Ausgabe' entwickelt hatte:

„Die Kunde der Hellenischen Alterthümer steht noch in ihren Anfängen; großer Stoff ist vorhanden, die meisten wissen ihn nicht zu gebrauchen. Wenige Gegenstände sind genügend abgehandelt, weil wer Einzelnes einigermaßen erschöpfen will, das Ganze kennen muß: ein Entwurf des Ganzen, mit wissenschaftlichem Geiste und umfassenden Ansichten gearbeitet, und nach festen Begriffen geordnet, nicht wie die bisherigen ein roher und unzusammenhängender Wust, nicht von einem Zusammenträger, sondern einem Forscher und Kenner, ist um so mehr ein Bedürfnis des gegenwärtigen Zeitalters, je mehr sich die Masse der Alterthumsgelehrten [...] in einer [...] meist auf das Geringfügigste gerichteten Sprachforschung [...] selbstgenügsam gefällt, [...] wodurch diejenigen, die [...] im Besitz der aus gebreitetsten Kunde sein sollten, [...] unsere Wissenschaft dem Leben und dem jetzigen Standpunkt der Gelehrsamkeit immer mehr entfremden ". 6

Gerade diese Kenntnis der Details und diese Weite des Blicks ließen Böckh aber nicht nur das Licht, sondern auch die Schattenseiten der griechischen Welt sehen:

„Wir verkennen nicht das Große und Erhabene in der Geschichte der Hellenen: wir geben zu, daß manches besser war als in unsern Staaten [...]; aber vieles war auch schlechter als das Unsrige. Nur die Einseitigkeit oder Oberflächlichkeit schaut überall Ideale im Alterthum; die Lobpreisung des Vergangenen und Unzufriedenheit mit der Mitwelt ist häufig bloß in einer Ver- 
stimmung des Gemüthes gegründet oder in Selbstsucht, welche die umgebende Gegenwart gering achtet, und nur die alten Heroen für würdige Genossen ihrer eingebildeten eigenen Größe hält. Es giebt Rückseiten, weniger schön als die gewöhnlich herausgekehrten [...] Die Hellenen waren im Glanze der Kunst und in der Blüthe der Freiheit unglücklicher als die meisten glauben".?

Aber Böckh erkannte zugleich, ,daß auch die Fehler mit Tugenden zusammenhingen und schwer entfernt werden konnten, wenn nicht die Freiheit, aller Tugenden Quell, gefährdet werden sollte" ${ }^{8}$ Und gerade, ,jenes rege Leben des Einzelnen, jene Freisinnigkeit und Großherzigkeit, jener unversöhnliche Haß gegen Unterdrückung und Knechtschaft und Willkür der Machthaber, die den Hellenen auszeichneten" ${ }^{\text {"69 }}$, sah er, so teuer sie auch bezahlt sein mochten, als erhaltenswerte Elemente des griechischen Lebens an, die auch modernen Staaten nicht fremd bleiben dürften, wenn diese sich nicht selbst aufgeben wollten. Weit über ihren Rang als altertumswissenschaftliche Publikation hinaus ist die 'Staatshaushaltung der Athener' auch ein bedeutendes Werk der Wirtschafts- und Rechtsgeschichte. Mit Recht hat der Volkswirtschaftler Wilhelm Roscher, der Begründer einer historischen Schule der Nationalökonomie, übrigens ein Schüler Böckhs, es in seiner 1874 erschienenen 'Geschichte der National-Ökonomik in Deutschland' als grundlegend für die geschichtliche Erforschung des Wirtschaftslebens bezeichnet. ${ }^{10}$

Schon bei den Vorarbeiten zur 'Staatshaushaltung der Athener' war Böckh die Bedeutung der griechischen Inschriften für die Behandlung seines Gegenstandes klar gewesen. So richtete er Anfang 1815, nicht lange nach seiner Aufnahme in die Königlich Preußische Akademie der Wissenschaften, an deren historisch-philologische Klasse einen Antrag auf die Erarbeitung eines Thesaurus Inscriptionum. Diesen Antrag begründete er $u$. a. damit, daß es Aufgabe einer Akademie sei, „Unternehmungen $\mathrm{zu}$ machen und Arbeiten zu liefern, welche kein Einzelner leisten kann, theils weil seine Kräfte denselben nicht gewachsen sind, theils weil ein Aufwand dazu erfordert wird, welchen kein 
Privatmann zu machen wagen wird“. ${ }^{11}$ Der Antrag wurde, vor allem von Philipp Buttmann tatkräftig unterstützt, von der Akademie angenommen, die daraufhin eine Kommission gründete, in der Böckh den Vorsitz übernahm und der außer ihm noch Niebuhr, Schleiermacher, Buttmann und Bekker angehörten. Zunächst sollten die griechischen Inschriften bearbeitet werden, und so erschien 1825 der erste Faszikel des schließlich nahezu zehntausend Inschriften umfassenden Corpus Inscriptionum Graecarum (CIG). Auf den Methodenstreit mit Gottfried Hermann, der sich an das Erscheinen dieses ersten Faszikels geknüpft hat, gehe ich hier nicht näher ein, da ich ihn in seiner Bedeutung für die Geschichte der Philologie an anderer Stelle ausführlich behandelt habe. ${ }^{12}$ Es mag genügen, darauf hinzuweisen, daß das griechische Corpus in dem von Theodor Mommsen begründeten Corpus Inscriptionum Latinarum sein lateinisches $\mathrm{Ge}$ genstück und in den Inscriptiones Graecae schließlich einen Nachfolger erhielt. Beide Corpora werden jetzt von der BerlinBrandenburgischen Akademie der Wissenschaften betreut, und unter Sachkennern ist es unbestritten, daß eine auf das Altertum gerichtete sozial-historische Forschung ohne die Heranziehung und Auswertung von zuverlässig edierten Inschriftentexten heute nicht mehr denkbar ist.

Die wissenschaftlichen Arbeiten von August Böckh stehen durchweg auf einem hohen Reflexionsniveau und zeichnen sich durch ein ungewöhnliches Methodenbewußtsein aus. Auch dieser Wesenszug Böckhs läßt sich auf seine Karlsruher Gymnasialzeit zurückverfolgen, in der ihn der an Leibniz und Locke anknüpfende Philosoph Gottlob August Tittel in Logik und Metaphysik unterrichtete. Das so geweckte Interesse fand weitere Nahrung in seinem Hallenser Platonstudium und insbesondere durch die enge Beziehung zu dem schon damals mit hermeneutischen Fragen beschäftigten Schleichermacher. Die Edition der eigenhändigen Manuskripte von Schleiermachers hermeneutischen Entwürfen durch Heinz Kimmerle hat uns die Entstehung seiner Hermeneutik besser verstehen gelehrt und läßt auch seinen Einfluß auf Böckh in neuem Lichte erscheinen. ${ }^{13}$ 
Im Sommersemester 1809 hielt der dreiundzwanzigjährige Böckh in Heidelberg zum ersten Mal eine Vorlesung über Enzyklopädie und Methodologie der philologischen Wissenschaften. In dieser Vorlesung, die er immer wieder durchgearbeitet und ergänzt und bis zum Sommersemester 1865, zwei Jahre vor seinem Tod, in regelmäßigen Abständen wiederholt hat und die von seinem Schüler Ernst Bratuscheck, auf Böckhs Vorlesungsheft und auf Kollegmitschriften gestützt, nach seinem Tode herausgegeben wurde, hat er sich am ausführlichsten über seine methodischen Prinzipien ausgesprochen. Aber auch in der praktischen philologischen Arbeit dringt er immer wieder auf methodische Besinnung. So schreibt er am 25. Juli 1820 an Friedrich Thiersch über die Pindarforschung seiner Zeit: „Nachdem nun in der Ausübung viel versucht ist und ich mich selbst genügsam versucht habe, halte ich es für zweckmäßig, über das vorhandene zu reflectiren, das heißt Grundsätze für die Kritik und Erklärung in einem einigermaßen theoretischen Zusammenhange darzustellen für diejenigen, welche nur immer das einzelne betrachten und keinen Totalüberblick gewinnen können." Und er schließt mit der Frage: „Glauben Sie nicht, daß das Methodische der Philologie, der eigentliche Kanon, Hermeneutik und Kritik, zu wenig zum Bewußtsein gebracht wird?" ${ }^{\star 14}$ Entsprechend erhebt er im Eingang seiner Abhandlung 'Ueber die kritische Behandlung der Pindarischen Gedichte' die Forderung,

,, daß [...] auch einmal wieder der Blick auf das [...] Methodische gerichtet werde, über welches noch wenig und nicht besonders eindringend gedacht ist. Denn die Meisten, welche sich mit dem Studium des Alterthums beschäftigen, haben kaum einen Begriff von dem innern Zusammenhange der verschiedenen Theile desselben, und von dem Wesen und Leben der dabei in Anwendung kommenden Thätigkeiten, sondern betreiben die Philologie mit einer gewissen Gedankenlosigkeit als ein gewohntes Geschäft oder eine Liebhaberei, höchstens von einem dunklen Gefühle der innern Vortrefflichkeit des Gegenstandes daran festgehalten ... "15

Eben diesem Mangel suchte Böckh mit seiner 'Encyklopädie und Methodologie der philologischen Wissenschaften' grundsätzlich 
abzuhelfen, in der er $\mathrm{u}$. a. rügte, daß man aus den bisherigen Begriffsbestimmungen der Philologie nicht erkenne, ,was Philologie ist oder sein sollte, sondern nur wie groß bei den Philologen der Mangel des Nachdenkens über ihr eigenes Studium ist" ${ }^{6}{ }^{16} \mathrm{Ich}$ kann auf dieses Werk hier nicht näher eingehen, und natürlich ist an ihm heute auch manches überholt. Aber wie immer man zu Böckhs (übrigens vielfach mißverstandener) eigener Bestimmung der eigentlichen Aufgabe der Philologie als „Erkennen des vom menschlichen Geist Producirten, d. h. des Erkannten" ${ }^{\text {"17 }}$ steht, in zweierlei Hinsicht scheint mir sein Werk eine fortdauernde Bedeutung zu besitzen: in der Forderung, daß eine jede wissenschaftliche Tätigkeit, von klar definierten Begriffen ausgehend, sich ihres methodischen Vorgehens bewußt zu sein hat, und in der Betonung der Notwendigkeit, auch das unscheinbarste Einzelphänomen im Rahmen eines größeren Ganzen zu sehen und seine Stellung innerhalb des Ganzen zu bestimmen.

In diesem Sinne entwarf Böckh im Zweiten Hauptteil seiner Enzyklopädie, der den materialen Disziplinen der Altertumslehre gewidmet war, sein eigenes System einer umfassenden Altertumswissenschaft, mit dem er dasjenige seines Lehrers Wolf nicht unwesentlich verfeinerte und ausbaute. Auf eine 'Allgemeine Alterthumslehre', die den Charakter des griechischen wie des römischen Altertums herauszuarbeiten sucht, folgen Abschnitte über das öffentliche und das private Leben der Griechen und Römer, über Religion und Kunst, Mythologie, Philosophie und Einzelwissenschaften sowie über Literatur und Sprache.

Aber so sehr Böckh auch auf methodische Reflexion und auf den Blick für das Ganze drang - im Mittelpunkt stand für ihn doch seine eigene Wissenschaft, die er als solche ganz ernst nahm, auch in ihrer Verantwortung gegenüber der Öffentlichkeit. Bei den zahlreichen Redeverpflichtungen, die ihm seine Ämter in Universität und Akademie, nicht zuletzt als Professor der Beredsamkeit, auferlegten, ergab sich immer wieder die Notwendigkeit, vor ein größeres Publikum zu treten. Er hat diese Verpflichtungen, wie eine Reihe von Selbstzeugnissen zeigt, nicht selten als eine Last empfunden, aber er hat sie immer wieder auf sich genom- 
men aus dem Gefühl der Verantwortung des Wissenschaftlers der Allgemeinheit gegenüber. Er vermochte auch verwickelte Sachverhalte anschaulich und leicht faßlich darzustellen, und so hat er zu einer großen Zahl von allgemeinen Fragen aus der Perspektive seines Faches heraus Stellung genommen: zum Verhältnis des Staates zum Unterrichtswesen, zur Umbildung der deutschen Universitäten, zum Verhältnis der Wissenschaft zur Praxis, zu den Pflichten des Wissenschaftlers, zu Berlin als Sitz der Wissenschaften (1860 anläßlich des Universitätsjubiläums) und zu vielem anderen mehr. Er tat es aus einer zutiefst liberalen Gesinnung und aus dem selbstbewußten Gefühl völliger Unabhängigkeit heraus - „Ich gehe meinen eigenen Weg und bin von Natur Protestant gegen alle menschliche Autorität" bekannte er einmal ${ }^{18}-$, aber immer um Vermittlung und Ausgleich bemüht. Gleich weit entfernt von selbstgenügsamer Forschung wie von einer Haltung, der das Altertum nicht mehr ist als ein Arsenal von Argumenten in den Auseinandersetzungen der Gegenwart, glaubte er es sich und seiner Zeit schuldig zu sein, die Ergebnisse seiner eindringenden Einzelforschung als Erkenntnispotential und heilsames Korrektiv in die Diskussion der Zeit einzubringen und damit für den Fortgang der Geschichte fruchtbar zu machen.

Damit gewann das Studium des Altertums eine Bedeutung, die weit über die Vermittlung von Einzelkenntnissen und die Einsicht in den Ablauf bestimmter historischer Prozesse hinausging. Gewiß hat die Altertumswissenschaft in den einhundertunddreißig Jahren, die seit dem Tode Böckhs vergangen sind, ihre führende Stellung im Kreis der Geisteswissenschaften eingebüßt und muß ihren Platz heute mit einer Fülle konkurrierender Wissenschaften teilen. Das hat seine Folgen natürlich auch für die Einschätzung der Bedeutung des griechisch-römischen Altertums für die $\mathrm{Ge}-$ genwart gehabt. Die Vorstellung, daß ein führender Naturwissenschaftler der Zeit die Vorlesungen eines ihm durch wissenschaftlichen Austausch eng verbundenen Altertumswissenschaftlers besucht, wie der alte Alexander von Humboldt das seinerzeit bei Böckh getan hat, mutet heute utopisch an. Aber man wird doch fragen müssen, ob wir es uns wirklich leisten können, auf all das zu verzichten, was die geistige und künstlerische Welt Griechen- 
lands und Roms an auch uns noch beschäftigenden Problemen durchdacht und gestaltet und an Möglichkeiten zur Lösung dieser Probleme entwickelt hat. August Böckh hat um diese Möglichkeiten gewußt und in seinem Werk immer wieder von ihnen gezeugt. „Wir sind Alle reicher geworden durch Böckh“, sagte Ernst Curtius in der Gedenkrede, die er am 24. November 1885 hier in Berlin anläßlich der 100. Wiederkehr des Geburtstages von Böckh gehalten hat, ${ }^{19}$ und das gilt, wie ich Ihnen zu zeigen versuchte, auch im Jahre 1997 noch. Aber ich meine, wir haben kein Recht, seiner zu gedenken, kein Recht auch, ein der Forschung und der Lehre dienendes Haus nach ihm zu benennen, ohne uns zugleich zu fragen, wie wir auf die Herausforderungen antworten, die von seinem Werk auch heute noch ausgehen. 


\section{Anmerkungen}

1 Zu Leben und Werk Böckhs vgl. insbesondere: M. Hoffmann, August Böckh. Lebensbeschreibung und Auswahl aus seinem wissenschaftlichen Briefwechsel, Leipzig 1901. Die wichtigste weitere Literatur bei: E. Vogt, Der Methodenstreit zwischen Hermann und Böckh und seine Bedeutung für die Geschichte der Philologie, in: Philologie und Hermeneutik im 19. Jahrhundert. Zur Geschichte und Methodologie der Geisteswissenschaften. Hrsg. von H. Flashar, K. Gründer, A. Horstmann, Göttingen 1979, S. 109, Anm.14. Seither vgl. vor allem: B. Schneider, August Boeckh. Altertumsforscher, Universitätslehrer und Wissenschaftsorganisator im Berlin des 19. Jahrhunderts. Ausstellung zum 200. Geburtstag ..., Wiesbaden 1985 (Staatsbibliothek Preußischer Kulturbesitz, Ausstellungskataloge 26) mit der dort S. 77 genannten Literatur; A. Horstmann, Antike Theoria und moderne Wissenschaft. August Boeckhs Konzeption der Philologie, Frankfurt a. M., Berlin, Bern, New York, Paris, Wien 1992 (Literatur S. 341-361). Böckh selbst hat seinen Namen stets mit Umlaut geschrieben, vgl. bereits Hoffmann, S. 2, Anm. 1; Vogt, S. 109, Anm. 14; Schneider, S. 9, Anm.

2 Dankbrief an das Lehrerkollegium des Lyceums zu Karlsruhe vom 29. Oktober 1860, vgl. Hoffmann (Anm. 1), S. 462-464. Das Zitat dort S. 463.

3 Th. Nipperdey, Deutsche Geschichte 1800-1866. Bürgerwelt und starker Staat, München 1983, S. 508 f.

4 A. Böckh, Die Staatshaushaltung der Athener, 1. Band, Berlin 1817, S. 128 (21851, S. 164; 31886, S. 148) im Hinblick auf das Zeugnis Athen. 264 C.

5 Staatshaushaltung I, S. 260 (2I, S. 342; 3I, S. 308).

6 Staatshaushaltung I, S. XIII f. (2I, S. XIII f.; 3I, S. XIX).

7 Staatshaushaltung II, S. 158 f. (2I, S. 791 f.; 3I, S. 710).

8 Staatshaushaltung II, S. 156 (2I, S. 789; 3I, S. 708).

9 Staatshaushaltung II, S. 159 (2I, S. 792; 3I, S. 711).

10 Vgl. Hoffmann (Anm. 1), S. 42.

11 Vgl. Schneider (Anm. 1), S. 30. Der Wortlaut des Antrags bei A. Harnack, Geschichte der Königlich Preußischen Akademie der Wissenschaften zu Berlin, Berlin 1900, Band 2, S. 374.

12 E. Vogt, Der Methodenstreit zwischen Hermann und Böckh und seine Bedeutung für die Geschichte der Philologie, in: Philologie und Hermeneutik im 19. Jahrhundert. Zur Geschichte und Methodologie der Geisteswissenschaften. Hrsg. von H. Flashar, K. Gründer, A. Horstmann, Göttingen 1979, S. 103-121.

13 Fr. D. E. Schleiermacher, Hermeneutik. Nach den Handschriften neu her 
ausgegeben und eingeleitet von $H$. Kimmerle, 2., verbesserte und erweiterte Auflage, Heidelberg 1974.

14 Der vollständige Brief bei Hoffmann (Anm. 1), S. 245f. Die beiden Zitate dort S. 246.

15 Gesammelte Kleine Schriften, 5. Band (Akademische Abhandlungen), Leipzig 1871, S. 248.

16 A. Böckh, Encyklopädie und Methodologie der philologischen Wissenschaften. Hrsg. von E. Bratuscheck, Leipzig 1877 (2. Aufl. 1886), S. 9.

17 Encyklopädie, S. 10.

18 Brief an den Theologen David Schulz vom 16. April 1809. Ein Auszug aus dem Brief bei Hoffmann (Anm. 1), S. 16f. Das Zitat dort S. 17.

19 E. Curtius, August Böckh, in: Alterthum und Gegenwart. Gesammelte Reden und Vorträge, 3. Band (Unter drei Kaisern), 2. vermehrte Auflage, Berlin 1895, S. 135-155. Das Zitat dort S. 154. 
